\title{
FOUR DIMENSIONAL TAUBERIAN THEOREMS VIA BLOCK DOMINATED MATRICES
}

\author{
RICHARD F. PATTERSON
}

\begin{abstract}
The goal of this paper is to present simple multidimmensional conditions to ensure that $T_{m, n}=\sum_{k, l=1,1}^{\infty, \infty} a_{m, n, k, l} x_{k, l}$ is included by P-convergence.
\end{abstract}

\section{Introduction}

The goal of this paper is to present simple conditions to ensure that $T_{m, n}=\sum_{k, l=1,1}^{\infty, \infty}$ $a_{m, n, k, l} x_{k, l}$ is included by $\mathrm{P}$-convergence. That is

$$
P-\lim _{k, l} x_{k, l}=P-\lim _{k, l} T_{k, l}
$$

whenever $P-\lim _{k, l} T_{k, l}$ exists. The method of proof to be used here is suggested by a argument employed by R. P. Agnew in [1]. R. P. Agnew uses two dimensional matrix transformation and single dimension sequences. In this paper the author will present a multidimensional analog of R. P. Agnew results.

\section{Definitions, Notations and Preliminary Results}

Definition 2.1. (Pringsheim, [6]). A double sequence $x=\left[x_{k, l}\right]$ has Pringsheim limit $L$ (denoted by $\mathrm{P}-\lim x=L$ ) provided that given $\epsilon>0$ there exists $N \in \mathbf{N}$ such that $\left|x_{k, l}-L\right|<\epsilon$ whenever $k, l>N$. We shall describe such an $x$ more briefly as "P-convergent".

Definition 2.2. (Pringsheim, [6]). A double sequence $x$ is called definitely divergent, if for every (arbitrarily large) $G>0$ there exist two natural numbers $n_{1}$ and $n_{2}$ such that $\left|x_{n, k}\right|>G$ for $n \geq n_{1}, k \geq n_{2}$.

Definition 2.3. (Patterson, [4]). The double sequence $[y]$ is a double subsequence of the sequence $[x]$ provided that there exist two increasing double index sequences $\left\{n_{j}\right\}$

Received and revised October 9, 2003.

2000 Mathematics Subject Classification. Primary 40 B05.

Key words and phrases. Pringsheim Limit Point, P-convergent, RH-regular. 
and $\left\{k_{j}\right\}$ such that if $z_{j}=x_{n_{j}, k_{j}}$, then $y$ is formed by

$$
\begin{array}{llll}
z_{1} & z_{2} & z_{5} & z_{10} \\
z_{4} & z_{3} & z_{6} & - \\
z_{9} & z_{8} & z_{7} & - \\
- & - & - & -
\end{array}
$$

Definition 2.4. (Patterson [4]). A number $\lambda$ is called a Pringsheim limit point of the double sequence $[x]$ provided that there exists a subsequence $[y]$ of $[x]$ that has Pringsheim limit $\lambda: \quad \mathrm{P}-\lim [y]=\lambda$.

With the definition of $\mathrm{P}$-convergence and the notion of subsequences presented in [4] it is clear that a double sequence $[x]$ is divergent in the Pringsheim sense (P-divergent) provided that $[x]$ is not $\mathrm{P}$-convergent. This is equivalent to the following: a double sequence $[x]$ is P-divergent if and only if either $[x]$ contains two subsequences with distinct finite limit points or $[x]$ contains an unbounded subsequence. Observe that, if $[x]$ contains an unbounded subsequence then $[x]$ also contains a definite divergent subsequence. In 1926 Robison presented a four dimensional analog of regularity for double sequences in which he added an additional assumption of boundedness: A four dimensional matrix $A$ is said to be RH-regular if it maps every bounded $\mathrm{P}$-convergent sequence into a $\mathrm{P}$ convergent sequence with the same P-limit. The necessary and sufficient conditions for RH-regularity of $(A x)_{m, n}=\sum_{k, l=1,1}^{\infty, \infty} a_{m, n, k, l} x_{k, l}$ are as follows:

$R H_{1}: \mathrm{P}-\lim _{m, n} a_{m, n, k, l}=0$ for each $k$ and $l$;

$R H_{2}: \mathrm{P}-\lim _{m, n} \sum_{k, l=1,1}^{\infty, \infty} a_{m, n, k, l}=1$;

$R H_{3}: \mathrm{P}-\lim _{m, n} \sum_{k=1}^{\infty}\left|a_{m, n, k, l}\right|=0$ for each $l$;

$R H_{4}: \mathrm{P}-\lim _{m, n} \sum_{l=1}^{\infty}\left|a_{m, n, k, l}\right|=0$ for each $k$;

$R H_{5}: \sum_{k, l=1,1}^{\infty, \infty}\left|a_{m, n, k, l}\right|$ is P-convergent; and

$R H_{6}$ : there exist finite positive integers $A$ and $B$ such that

$\sum_{k, l>B}\left|a_{m, n, k, l}\right|<A$.

The following definition for Pringsheim limit superior and inferior is presented [5]: Let $[x]=\left\{x_{k, l}\right\}$ be a double sequence of real numbers and for each $n$, let $\alpha_{n}=\sup _{n}\left\{x_{k, l}\right.$ : $k, l \geq n\}$. The Pringsheim limit superior of $[x]$ is defined as follows:

(1) if $\alpha=+\infty$ for each $\mathrm{n}$, then $\mathrm{P}-\lim \sup [x]:=+\infty$;

(2) if $\alpha<\infty$ for some $n$, then P-limsup $[x]:=\inf _{n}\left\{\alpha_{n}\right\}$.

Similarly, let $\beta_{n}=\inf _{n}\left\{x_{k, l}: k, l \geq n\right\}$ then the Pringsheim limit inferior of $[x]$ is defined as follows:

(1) if $\beta_{n}=-\infty$ for each $\mathrm{n}$, then $\mathrm{P}-\liminf [x]:=-\infty$;

(2) if $\beta_{n}>-\infty$ for some $n$, then P-liminf $[x]:=\sup _{n}\left\{\beta_{n}\right\}$. 


\section{Main Results}

Definition 3.1. A four dimensional matrix $A$ is said to $\left\{B_{m, n}^{A}(\zeta, \eta)\right\}$-dominated if $\zeta \& \eta$ are nonnegative interges and

$$
P-\liminf _{m, n}\left\{\left|\sum_{(k, l) \in B_{m, n}^{A}(\zeta, \eta)} a_{m, n, k, l}\right|-\sum_{(k, l) \notin B_{m, n}^{A}(\zeta, \eta)}\left|a_{m, n, k, l}\right|\right\}>0
$$

where

$$
B_{m, n}^{A}(\zeta, \eta)=\left\{(k, l): A=\left[a_{m, n, k, l}\right], m-\zeta<k \leq m+\zeta, \& n-\eta<l \leq n+\eta\right\} .
$$

Theorem 3.1. If $A$ is an RH-regular real matrix that is $\left\{B_{m, n}^{A}(\zeta, \eta)\right\}$-dominated and $[x]$ is a bounded double sequence such that $[A x]$ is P-convergent then $[x]$ is P-convergent.

Proof. Suppose $[x]$ is a bounded real double sequence which is divergent in the Pringsheim sense. We shall use this sequence to show that there does not exists $r$ real such that $P-\lim (A x)_{m, n}=r$. Let $R=P-\lim \sup _{k, l}\left|x_{k, l}-r\right|$. The regularity condition $\mathrm{RH}_{2}$ implies the following:

$$
(A x)_{m, n}-r=o(1)+\sum_{k, l=1,1}^{\infty, \infty} a_{m, n, k, l}\left(x_{k, l}-r\right) .
$$

Suppose $0<\epsilon<R$ and choose $k_{0}$ and $l_{0}$ large such that for $k>k_{0}$ and $l>l_{0}$ implies $\left|x_{k, l}-r\right|<R+\epsilon$. This yields the following:

$$
\begin{aligned}
\left|(A x)_{m, n}-r\right| \geq & o(1)+\left|\sum_{(k, l) \in B_{m, n}^{A}(\zeta, \eta)} a_{m, n, k, l}\left(x_{k, l}-r\right)\right| \\
& -\sum_{(k, l) \notin B_{m, n}^{A}(\zeta, \eta)}\left|a_{m, n, k, l}\right|\left|x_{k, l}-r\right| \\
\geq & o(1)+\left|\sum_{(k, l) \in B_{m, n}^{A}(\zeta, \eta)} a_{m, n, k, l}\left(x_{k, l}-r\right)\right| \\
& -\sum_{(k, l) \notin B_{m, n}^{A}(\zeta, \eta) \& k \geq k_{0}, l \geq l_{0}}\left|a_{m, n, k, l}\right|\left|x_{k, l}-r\right| \\
> & o(1)+\left|\sum_{(k, l) \in B_{m, n}^{A}(\zeta, \eta)} a_{m, n, k, l}\left(x_{k, l}-r\right)\right| \\
& -(R+\epsilon) \sum_{(k, l) \notin B_{m, n}^{A}(\zeta, \eta) \& k \geq k_{0}, l \geq l_{0}}\left|a_{m, n, k, l}\right| \\
\geq & o(1)+\left|\sum_{(k, l) \in B_{m, n}^{A}(\zeta, \eta)} a_{m, n, k, l}\left(x_{k, l}-r\right)\right| \\
& -R \sum_{(k, l) \notin B_{m, n}^{A}(\zeta, \eta) \& k \geq k_{0}, l \geq l_{0}}\left|a_{m, n, k, l}\right|-\epsilon\|A\|
\end{aligned}
$$


where $\|A\|=\sup _{m, n} \sum_{k, l=1,1}^{\infty, \infty}\left|a_{m, n, k, l}\right|$. We can now assume that there are infinitely many $m$ and $n$ such that for $(k, l) \in B_{m, n}^{A}(\zeta, \eta)$ we obtain the following $\left|x_{k, l}-r\right|>R-\epsilon$. Thus

$$
\begin{aligned}
& \left|(A x)_{m, n}-r\right|>o(1)+\left|\sum_{(k, l) \in B_{m, n}^{A}(\zeta, \eta)} a_{m, n, k, l}\left(x_{k, l}-r\right)\right| \\
& -R \sum_{(k, l) \notin B_{m, n}^{A}(\zeta, \eta) \& k \geq k_{0}, l \geq l_{0}}\left|a_{m, n, k, l}\right|-\epsilon\|A\| \\
& \geq o(1)+(R-\epsilon)\left|\sum_{(k, l) \in B_{m, n}^{A}(\zeta, \eta)} a_{m, n, k, l}\right| \\
& -R \sum_{(k, l) \notin B_{m, n}^{A}(\zeta, \eta) \& k \geq k_{0}, l \geq l_{0}}\left|a_{m, n, k, l}\right|-\epsilon\|A\| \\
& \left|(A x)_{m, n}-r\right|>o(1)-\epsilon\left\{\|A\|+\left|\sum_{(k, l) \in B_{m, n}^{A}(\zeta, \eta)} a_{m, n, k, l}\right|\right\} \\
& +R\left\{\left|\sum_{(k, l) \in B_{m, n}^{A}(\zeta, \eta)} a_{m, n, k, l}\right|-\sum_{(k, l) \notin B_{m, n}^{A}(\zeta, \eta)}\left|a_{m, n, k, l}\right|\right\} \\
& \geq o(1)-2 \epsilon\|A\|+R\left\{\left|\sum_{(k, l) \in B_{m, n}^{A}(\zeta, \eta)} a_{m, n, k, l}\right|-\sum_{(k, l) \notin B_{m, n}^{A}(\zeta, \eta)}\left|a_{m, n, k, l}\right|\right\} .
\end{aligned}
$$

Since $\epsilon$ is an arbitrarily small positive number, we obtain the following

$$
P-\liminf _{m, n}\left|(A x)_{m, n}-r\right|>0 .
$$

Thus $[A x]$ diverges in the Pringsheim sense.

Theorem 3.2. Suppose $A$ is a four dimensional summability matrix method and $[x]$ is unbounded double sequence for which $(A x)_{m, n}=\sum_{k, l=1,1}^{\infty, \infty} a_{m, n, k, l} x_{k, l}$ exist for each $m$ and $n$ and $[x]$ contains an unbounded subsequence (i.e. unbounded in the Pringsheim sense). Then $(A x)_{m, n}$ is unbounded in the Pringsheim sense.

Proof. Suppose $[x]$ is unbounded in the Pringsheim sense then there are infinity many pairs $(m, n)$ in $B_{m, n}^{A}(\zeta, \eta)$ such that

$$
x_{k, l} \leq x_{m, n} \text { for }(k, l) \notin B_{m, n}^{A}(\zeta, \eta) .
$$

For pairs of $(m, n)$ satisfying the above condition we obtain the following:

$$
\left|(A x)_{m, n}\right|=\left|\sum_{(k, l) \in B_{m, n}^{A}(\zeta, \eta)} a_{m, n, k, l}+\sum_{(k, l) \notin B_{m, n}^{A}(\zeta, \eta)} a_{m, n, k, l}\right|
$$




$$
\begin{aligned}
& \geq\left|\sum_{(k, l) \in B_{m, n}^{A}(\zeta, \eta)} a_{m, n, k, l} x_{k, l}\right|-\sum_{(k, l) \notin B_{m, n}^{A}(\zeta, \eta)}\left|a_{m, n, k, l}\right|\left|x_{k, l}\right| \\
& \geq\left[\left|\sum_{(k, l) \in B_{m, n}^{A}(\zeta, \eta)} a_{m, n, k, l}\right|-\sum_{(k, l) \notin B_{m, n}^{A}(\zeta, \eta)}\left|a_{m, n, k, l}\right|\right]\left|x_{m, n}\right| .
\end{aligned}
$$

Hence $P-\lim \sup _{m, n}\left|(A x)_{m, n}\right|=\infty$. Thus $(A x)_{m, n}$ is unbounded in the Pringsheim sense.

\section{References}

[1] R. P. Agnew, Equivalence of methods for evaluation of sequences, Proc. Amer. Math. Soc. 3(1952), 550-556.

[2] H. J. Hamilton, Transformations of multiple sequences, Duke Math. Jour. 2(1936), 29-60.

[3] G. H. Hardy, Divergent Series, Oxford Univ. Press, London. 1949.

[4] R. F. Patterson, Analogues of some fundamental theorems of summability theory, internat. J. Math. \& Math. Sci. 23(2000), 1-9.

[5] R. F. Patterson, Double sequence core theorems, internat. J. Math. \& Math. Sci. 22(1999), 785-793.

[6] A. Pringsheim, Zur theorie der zweifach unendlichen zahlenfolgen, Mathematische Annalen 53(1900), 289-321

[7] G. M. Robison, Divergent double sequences and series, Amer. Math. Soc. trans. 28(1926), 50-73. Stanislaw Mazur and Wladyslaw Orlicz, Sur les méthodes linéaires de sommation, C. R. Acad. Sci. Paris 196(1933), 32-34.

Department of Mathematics and Statistics, University of North Florida, building 11 Jacksonville, Florida, 32224.

E-mail: rpatters@unf.edu 